\title{
Power-Efficient Joint Compressed Sensing of Multi-Lead ECG Signals
}

\author{
Hossein Mamaghanian, Giovanni Ansaloni, David Atienza and Pierre Vandergheynst \\ School of Engineering, Ecole Polytechnique Fédérale de Lausanne (EPFL) \\ Email: name.surname@epfl.ch
}

\begin{abstract}
Compressed Sensing (CS) is a new acquisitioncompression paradigm for low-complexity energy-aware sensing and compression. By merging both sampling and compression, CS is very promising to develop practical ultra-low power readout systems for wireless bio-signal monitoring devices, where large amounts of sensor data need to be transferred through power-hungry wireless links.

Lately CS has been successfully applied for real-time energyaware single-lead ECG compression on resource-constrained Wireless Body Sensor Network (WBSN) motes [1]. Building on our previous work, in this paper we propose a new and promising approach for joint compression of multi-lead ECG signals, where strong correlations exist between them. This situation that exhibit strong correlations, can be exploited to reduce even further amount of data to be transmitted wirelessly, thus addressing the important challenge of ultra-low-power embedded monitoring of multi-lead ECG signals.
\end{abstract}

\section{INTRODUCTION}

$\mathrm{CS}$ is a new sensing and processing paradigm, which challenges the traditional analog-to-digital conversion based on the Shannon sampling theorem. For sparse signals such as the electrocardiogram (ECG), Nyquist-rate sampling produces a large amount of redundant digital samples, which are costly to wirelessly transmit in the context of our target mobile ECG monitoring systems, and require to be further compressed using non-linear digital techniques. CS is a methodology that has been recently proposed to address this problem.

Capitalizing on this sparsity, we have recently proposed [1], [2], to apply the emerging compressed sensing (CS) approach [3] for a low-complexity, real-time and energy-efficient ECG signal compression on WBSN motes. We have also quantified the potential of compressed sensing (CS) for lowcomplexity energy-efficient ECG compression on the stateof-the-art Shimmer ${ }^{\mathrm{TM}}$ WBSN mote. Interestingly, our results show that CS represents a competitive alternative to state-ofthe-art digital wavelet transform (DWT)-based ECG compression solutions in the context of WBSN-based ECG monitoring systems. The results validate the suitability of compressed sensing for real-time energy-aware ECG compression on resource-constrained WBSN motes.

Nonetheless, in a real scenario many of the bio-signals are acquired on multiple channels. In these cases, sampling, compressing and reconstructing each signal individually is clearly sub-optimal, because leads are not independent sources, and are in fact strongly correlated. In the case of the ECG acquisitions, all signals can be considered as different projections of a single multidimensional source, which is the electrical field produced by the heart. Thus not only these leads are not independent, but each lead conveys useful information about other leads.

In this paper, the mutual information between the leads of bio-signals can be exploited to optimize CS-based compression, considering the example of multi-lead ECG acquisitions. We also propose techniques to exploit this information for optimized recovery of the joint compressed sensing. This optimization will directly translate to less measurement needed for the recovery, leading to reduction in transmission bandwidth (and, ultimately, of power consumption) without quality degradation.

Notation: In all the following, normal letters designate scalar quantities, boldface lower-case letters indicate column vectors, and boldface capitals represent matrices. Moreover, $m_{i}$ and $\mathbf{M}_{i, j}$ are the $i^{t h}$ entry of vector $\mathbf{m}$ and the $(i, j)^{t h}$ entry of matrix $\mathbf{M}$, respectively. Finally, $(.)^{H}$ and $\|.\|_{p}$ denote the conjugate transpose, and the $\ell_{p}$-norm of a vector, respectively.

\section{COMPRESSED SENSING AND SPARSE RECOVERY}

Let $\mathbf{x}$ be the real-valued $N$-dimensional ECG signal vector $\left(\mathrm{x} \in \mathbb{R}^{N}\right)$. The original ECG signal $\mathrm{x}$ has a sparse approximation, i.e., it can be represented by a linear superposition of $S$ elements of an orthonormal wavelet basis, $\mathbf{x} \approx \sum_{k=1}^{S} \alpha_{k} \boldsymbol{\psi}_{k}$, with $S \ll N$. Conventionally, one would collect ECG samples at the Nyquist rate forming $\mathbf{x}$ and then compress it using non-linear digital compression techniques. CS offers a striking alternative by showing that you can collect roughly $S$ samples using simple analog measurement waveforms, thus sensing/sampling and compressing at the same time (Analog CS) [4]. But in present work CS is done after ADC (digital $\mathrm{CS}$ ), where digital samples vector $\mathbf{x}$ are available. Thus compressed data vector $\mathbf{y} \in \mathbb{R}^{M}$ is represented by $\mathbf{y}=\boldsymbol{\Phi} \mathbf{x}$, where $\boldsymbol{\Phi} \in \mathbb{R}^{M \times N}$ is called sensing matrix.

It is important to notice that the sensing matrix $\boldsymbol{\Phi}$ does not depend on the signal: CS proposes a simple linear sampling strategy that is only marginally off the optimal but complex best adaptive strategy. To guarantee the robust and efficient recovery of the $S$-sparse signal $\alpha_{S}$, the sensing matrix $\boldsymbol{\Phi}$ must obey the key restricted isometry property (RIP) [5]:

$$
\left(1-\delta_{S}\right)\|\boldsymbol{\alpha}\|_{2} \leq\|\boldsymbol{\Phi} \boldsymbol{\Psi} \boldsymbol{\alpha}\|_{2} \leq\left(1+\delta_{S}\right)\|\boldsymbol{\alpha}\|_{2},
$$

for all $S$-sparse vectors $\boldsymbol{\alpha} . \delta_{S}$ is the isometry constant of matrix $\boldsymbol{\Phi}$, which must be not too close to one. In CS literature, i.i.d sub-Gaussian matrices are popular, since they are known 
to be measurements optimal. The lower bound for the number of measurements is proven to be $M=\Omega(S \log N)$. But as far as sparsity $S$ considered, Gaussian matrices are not space optimal and need a huge storage space and nor time optimal since complexity of the encoding and reconstruction is in order of $\mathcal{O}(M N)$, which makes them far from being practical and real-time for a limited resource WBSNs. As an alternative, here sparse binary matrix is used as our sensing matrix $\boldsymbol{\Phi}$, where each column contain only $d$ nonzero elements equal to $1,(d \ll M \ll N)$ [1] with required space and time complexity of $\mathcal{O}(d N)$. For such a sensing matrix, the RIP property of 1 is not valid. However, it satisfies a different form of this property, with slightly more required measurements [6] which guarantees the reconstruction.

If RIP holds, then an approximate sparse signal reconstruction can be accomplished by solving the following convex optimization problem:

$$
\min _{\tilde{\boldsymbol{\alpha}} \in \mathbb{R}^{N}}\|\tilde{\boldsymbol{\alpha}}\|_{1} \quad \text { subject to } \quad\|\boldsymbol{\Phi} \boldsymbol{\Psi} \tilde{\boldsymbol{\alpha}}-\mathbf{y}\|_{2} \leq \sigma,
$$

where $\sigma$ bounds the amount of noise corrupting the data. This is a convex optimization problem that can be solved with a variety of techniques. Later in Section III we discuss one particular algorithm that does so.

\section{Multi LEAD ECG AND JOINT COMPRESSION}

The CS sampling and reconstruction steps has been described in previous Section II. In this section we will discuss the problem of the multileads ECG signals and their joint compression. Let $\mathbf{X} \in \mathbb{R}^{N \times L}$ be the real valued matrix of ECG signals where each column corresponds to a single ECG leads. Now the CS problem could be written as $\mathbf{Y}=\boldsymbol{\Phi} \mathbf{X}$, where $\boldsymbol{\Phi}$ is the sensing matrix. Let's imagine that $\mathrm{X}=\boldsymbol{\Psi} \mathbf{A}$ is the matrix of the wavelet representation of the original ECG matrix: $\mathbf{A} \in \mathbb{R}^{N \times L}=\left[\boldsymbol{\alpha}_{1}, \boldsymbol{\alpha}_{2}, \ldots, \boldsymbol{\alpha}_{L}\right]$, and $\boldsymbol{\alpha}_{i}, i \in\{1,2, \ldots, L\}$, is the vector of wavelet coefficients of each ECG lead. We could rewrite the problem (2) in the form:

$$
\min _{\alpha \in \mathbb{R}^{N}} f(\boldsymbol{\alpha})+\lambda g(\boldsymbol{\alpha})
$$

where $f: \mathbb{R}^{N} \rightarrow \mathbb{R}$ is a convex differentiable function and $g: \mathbb{R}^{N} \rightarrow \mathbb{R}$ is a sparsity-inducing norm. Here, our data-fitting function is $f(\boldsymbol{\alpha})=\|\boldsymbol{\Phi} \boldsymbol{\Psi} \tilde{\boldsymbol{\alpha}}-\mathbf{y}\|_{2}$ and usually in context of the CS reconstruction $\ell_{1}$ norm is used to enforce sparsity. In case of the multi-lead ECG compression, where there is a strong correlation between the sparsity structure among the leads, and the sparse coefficients model should be refine to take it into account. In such a situation, where nonzero coefficients are naturally partitioned in subsets or groups, the best choice could be using a group-sparsity inducing term [7]. Figure 1, shows the support of the best $S$-term approximation of the investigated multi-lead ECG recordings. It shows that support set of the $S$-term approximation for leads are very similar, so that a joint sparse support selection can be performed in the reconstruction algorithm. We propose to achieve this by replacing the traditional $\ell_{1}$ norm with the following $\ell_{1} / \ell_{2}$ group prior: $g(\boldsymbol{\alpha})=\sum_{g \in \mathcal{G}}\left\|\boldsymbol{\alpha}_{g}\right\|_{2}$. where $\mathcal{G}$ is a partition

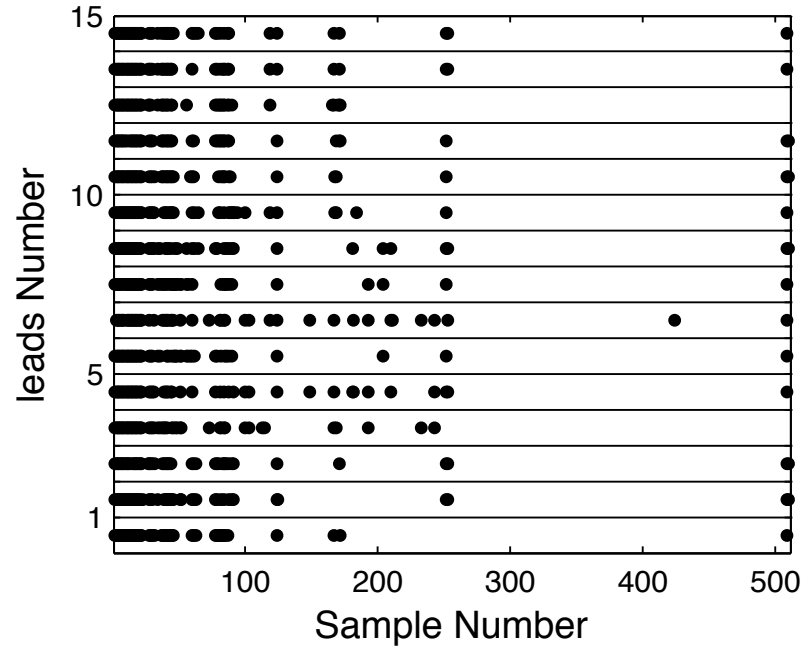

Fig. 1: Support of the best S-term approximation of multi-lead ECG signals.

of $\{1, \ldots, p\}$, and $\boldsymbol{\alpha}_{g}$ denotes the vector in $\mathbb{R}^{|g|}$ recording the coefficient of $\boldsymbol{\alpha}$ indexed by $g$ in $\mathcal{G}$. This is known as a mixed $\ell_{1} / \ell_{2}$-norm. It behaves like an $\ell_{1}$-norm on the vector $\left(\left\|\alpha_{g}\right\|_{2}\right)_{g \in \mathcal{G}}$ in $\mathbb{R}^{|\mathcal{G}|}$, and therefore, $g$ induces group sparsity.

The CS problem (3) can be cast as a second order cone programming problem and thus could be solved via interior point methods [8]. However, it involves dense matrices, which often precludes the use and potential advantage of sophisticated interior point methods. Here, we use the class of proximal methods, which involves very cheap computational effort compared to interior point methods. This class of methods could be applied to solve a problem of the form (3), where the problem is as the sum of a generic smooth differentiable function $f$ with Lipschitz-continuous gradient, and a non-differentiable function $\lambda g($.$) . Here we use the Fast Iterative soft thresholding$ algorithm (FISTA) for the $\ell_{1}$-norm method [9].

\section{RESUlts}

To validate the performance of the proposed joint compression scheme, we use the PTB Diagnostic ECG Database [10], available on the physionet website. The database contains 549 records of 15 -lead ECG from 290 subjects. Signals are sampled at $1 \mathrm{KHz}$ with 16 -bit resolution.

Moreover, to quantify the compression performance while assessing the diagnostic quality of the compressed ECG records, we employ the two most widely used performance metrics, namely the compression ratio $(C R)$ and percentage root-mean-square difference $(P R D)$. $C R$ is defined as: $C R=\frac{b_{\text {orig }}-b_{\text {comp }}}{b_{\text {orig }}} \times 100$, where $b_{\text {orig }}$ and $b_{\text {comp }}$ represent the number of bits required for the original and compressed signals, respectively. The $P R D$, and associated signal-to-noise ratio $(S N R)$, quantifies the percent error between the original signal vector $\mathbf{x}$ and the reconstructed $\tilde{\mathbf{x}}$ :

$$
\begin{aligned}
P R D & =\frac{\|\mathbf{x}-\tilde{\mathbf{x}}\|_{2}}{\|\mathbf{x}\|_{2}} \times 100, \\
S N R & =-20 \log _{10}(0.01 P R D) .
\end{aligned}
$$




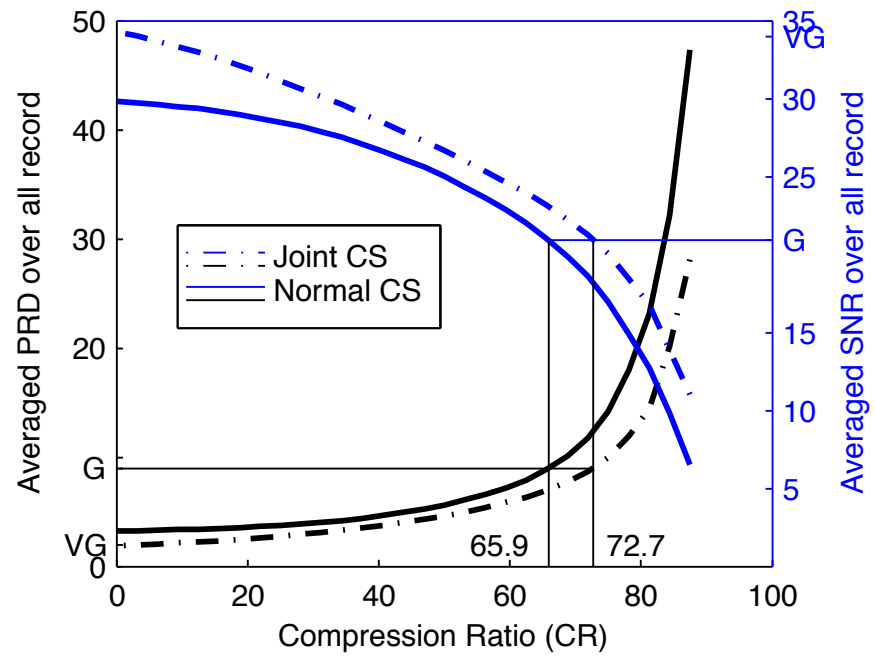

Fig. 2: Output averaged PRD (left) and SNR (right) over all records for different compression ratios

The link between the measured PRD and the diagnostic distortion is established based on the work of Zigel et al. on the weighted diagnostic measure for ECG signal compression [11], which classifies the different values of PRD based on the signal quality perceived by a specialist. Accordingly, the reconstruction with PRD value between $0 \sim 2 \%$ and $2 \sim 9 \%$ categorized as "very good" and "good" quality. For higher PRD values it is not possible to determine the quality group.

Here, in this section we present the results for both Normal $\mathrm{CS}$ reconstruction with $\ell_{1}$ regularization per channel and joint group reconstruction with $\ell_{1} / \ell_{2}$-norm. In all our experiments ECG matrix $\mathrm{X}$ is a window of size $N=512$ and $L=15$. For sensing matrix $\boldsymbol{\Phi}$, sparse random binary matrix is used, where each column contain only $d$ nonzero elements $(d=35)$ [12]. The results are averaged over 100 packets randomly selected from all the database records.

Figure 2 compares the output SNR and PRD, averaged over all database records, for both algorithms and different compression ratios. The "Normal reconstruction" and "Joint reconstruction" correspond to convex minimization with $\ell_{1}$ and $\ell_{1} / \ell_{2}$ constrains respectively. This figure shows the average PRD and SNR, but there is a large variance across individual records. Alternatively, Figure 3 shows the box plots for both algorithms. On each box, the central mark is the median, the edges of the box are the $25^{\text {th }}$ and $75^{\text {th }}$ percentiles, and the whiskers extend to the most extreme. In both figures the level corresponding to the "good" (corresponding to PRD level between $2 \%$ and $9 \%$ ) and "very good" (PRD level below than $2 \%$ reconstruction qualities are indicated. It shows that the joint reconstruction clearly outperforms the normal CS reconstruction for all compression ratios: "good" reconstruction quality for Normal CS can be reached with $65.9 \%$ of compression, while for joint reconstruction this number could increase close to $7 \%$ to reach up to $72.7 \%$. As noted in our previous work [1], compression can be further enhanced by removing inter-packet redundancy and performing Huffman
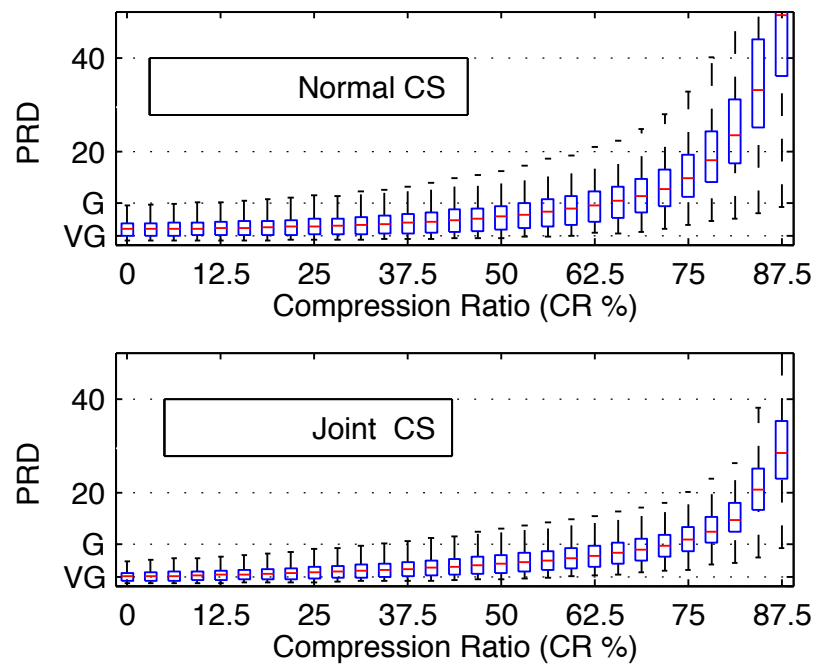

Fig. 3: Box plots for all database records for Normal reconstruction (top) and Joint reconstruction (bottom)

coding. The results of our work shows that compression level could be enhanced by close to $50 \%$ by removing the interpacket redundancy and Huffman coding without scarifying the reconstruction quality.

Figures $4 \mathrm{a}$ and $4 \mathrm{~b}$ compare the averaged SNR for each lead for different compressions. The output SNR are color coded. These figures also show that joint reconstruction enhances the quality of all leads at the same time. The weak behavior for leads number 4 and 7 , compared to other leads are due to the fact that these leads are very noisy compared the rest of the leads. This is also shown on the Normal reconstruction in Figure $4 \mathrm{a}$.

\section{ENERgY CONSUMPTION ANALYSIS}

In this section, we further investigate the power consumption of the both algorithms. Our experimental setup consists of a Shimmer wireless node[13] implementing embedded ECG compression by CS and wirelessly transmitting the compressed data using its IEEE 802.15.4-compliant radio to a remote base station. A simple medium access control (MAC) scheme is implemented between the node and the base station in FreeRTOS: the base station periodically sends a beacon to maintain node synchronization, and the ECG sensing node sends its data in its guaranteed time slots (GTS) which is assigned by the base station and notified in the beacon. This simple MAC is in fact compatible with the beacon-enabled mode of the IEEE 802.15.4 MAC protocol [14].

To study the power consumption of the different working modes of the node was characterized. Our measurements show an average node power consumption of $84.6 \mathrm{~mW}$ during radio reception, and of $64.35 \mathrm{~mW}$ during transmission. The radio is only turned on for beacon reception (i.e., $2.7 \mathrm{~ms}$ ), and when there is a data packet to be transmitted. Moreover, each transmitted packet is 127-Byte long with 13 Byte of MAC overhead $(4.2 \mathrm{~ms})$. The rest of time, the radio is off and the processor is idle and only wakes up for sampling to preserve 


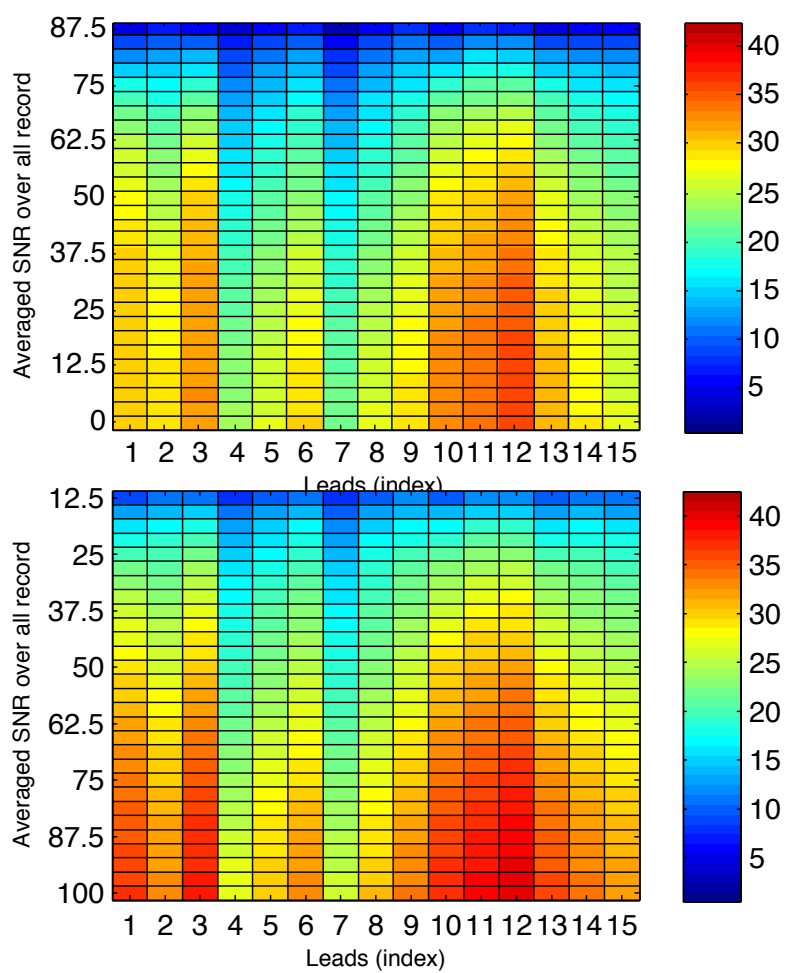

Fig. 4: Averaged SNR for all the leads for different compression levels for normal CS (top) and joint reconstruction (bottom)

energy, which corresponds to an average power consumption of $6.44 \mathrm{~mW}$. Figure 5 , shows the breakdown of the total energy consumption for both algorithms corresponding to "good" reconstruction quality, between the three main processes: 1) the wireless communication; 2) the code execution on the $\mathrm{CPU}$; and 3) the sampling and the operation system (OS). As shown in Figure 5, most of the energy is due to the radio link which strongly recommends the need for a suitable compression technique. Total energy consumption for the normal CS and Joint compression are $41.3 \mu \mathrm{J}$, and $32.7 \mu \mathrm{J}$ respectively. Our results shows that Joint reconstruction could reach up to $22 \%$ reduction in overall energy consumption of the node. This will increase overall lifetime of the node by $26 \%$ from 25 hours to 31.7 hours on a $280 m A h @ 3.7 \mathrm{~V}$ battery.

\section{CONCLUSION}

This paper presents a novel algorithm for compression of the multi-lead ECG signals. Exploiting the strong correlation between different leads, we could enhance the overall compression rate by $7 \%$ compared to Normal CS reconstruction, where no additional coding is done except CS. Our experiments on a test setup based on Shimmer wireless nodes show that this could reduce the overall power consumption of the node by $22 \%$ and overall lifetime of the node by $26 \%$. As such, our results validate the suitability of joint compressed sensing for real-time energy-aware multi-lead ECG compression.

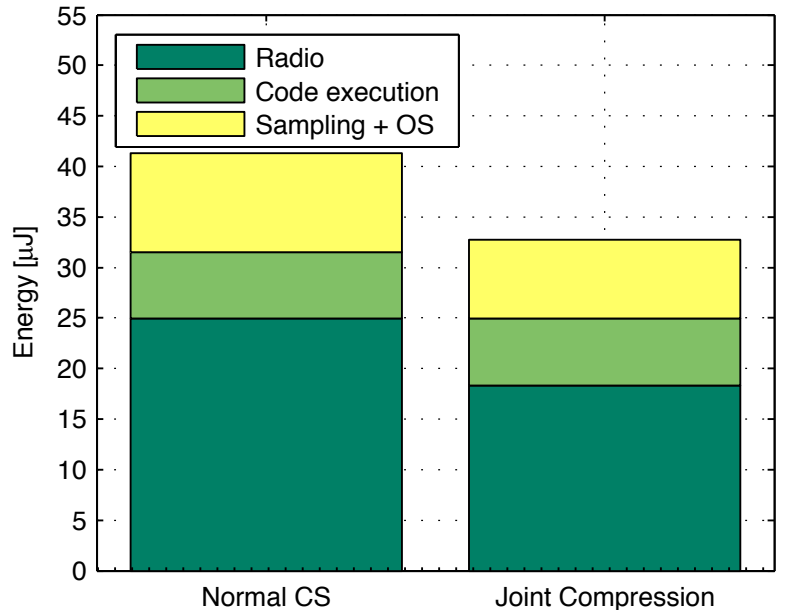

Fig. 5: Breakdown of energy consumption of Shimmer

\section{REFERENCES}

[1] H. Mamaghanian, N. Khaled, D. Atienza, and P. Vandergheynst, "Compressed sensing for real-time energy-efficient ecg compression on wireless body sensor nodes," Biomedical Engineering, IEEE Transactions on, vol. 58, no. 9, pp. $2456-2466$, sept. 2011.

[2] — , "Structured sparsity models for compressively sensed electrocardiogram signals: A comparative study," in Biomedical Circuits and Systems Conference (BioCAS), 2011 IEEE, Nov 2011, pp. 125-128.

[3] D. Donoho, "Compressed sensing," Information Theory, IEEE Transactions on, vol. 52, no. 4, pp. 1289 -1306, april 2006.

[4] H. Mamaghanian, N. Khaled, D. Atienza, and P. Vandergheynst, "Design and exploration of low-power analog to information conversion based on compressed sensing," Emerging and Selected Topics in Circuits and Systems, IEEE Journal on, vol. 2, no. 3, pp. 493-501, 2012.

[5] E. Candes, J. Romberg, and T. Tao, "Stable signal recovery from incomplete and inaccurate measurements," Communications on Pure and Applied Mathematics, vol. 59, pp. 1207-1223, 2006.

[6] V. Chandar, "A negative result concerning explicit matrices with the restricted isometry property," Tech. Rep., 2008.

[7] M. Kowalski and B. Torrésani, "Sparsity and persistence: mixed norms provide simple signal models with dependent coefficients," Signal, Image and Video Processing, vol. 3, no. 3, pp. 251-264, 2009. [Online]. Available: http://dx.doi.org/10.1007/s11760-008-0076-1

[8] Y. Nesterov, A. S. Nemirovskii, and Y. Ye, Interior-point polynomial algorithms in convex programming. SIAM, 1994, vol. 13.

[9] A. Beck and M. Teboulle, "A fast iterative shrinkage-thresholding algorithm with application to wavelet-based image deblurring," in Acoustics, Speech and Signal Processing, 2009. ICASSP 2009. IEEE International Conference on, april 2009, pp. $693-696$.

[10] A. L. Goldberger, L. A. Amaral, L. Glass, J. M. Hausdorff, P. C. Ivanov, R. G. Mark, J. E. Mietus, G. B. Moody, C.-K. Peng, and H. E. Stanley, "Physiobank, physiotoolkit, and physionet components of a new research resource for complex physiologic signals," Circulation, vol. 101, no. 23 , pp. e215-e220, 2000.

[11] Y. Zigel, A. Cohen, and A. Katz, "The weighted diagnostic distortion (wdd) measure for ecg signal compression," Biomedical Engineering, IEEE Transactions on, vol. 47, no. 11, pp. $1422-1430$, nov. 2000.

[12] H. Mamaghanian, N. Khaled, and P. Vandergheynst, "A real-time compressed sensing-based personal electrocardiogram monitoring system," in IEEE International Symposium on Circuits and Systems ISCAS' 11, Oct. 2010.

[13] Shimmer Research, "Shimmer Small Wireless Sensor Platform Designed to Support Wearable Applications." [Online]. Available: http://shimmer-research.com

[14] IEEE, "Wireless medium access control (MAC) and physical layer (PHY) specifications for low-rate wireless personal area networks (LRWPANs)," IEEE, Tech. Rep. IEEE 802.15.4 Std., 2003. 